\title{
Importancia de la nutrición en pacientes adultos mayores con infección por CoviD-19
}

\author{
Importance of nutrition in older adult patients with the COVID-19 infection
}

Importância da nutrição em pacientes idosos infectados por COVID-19

\author{
María del Consuelo Velázquez-Alva'; María Fernanda Cabrer Rosales²; María Esther Irigoyen Camacho³ \\ 1 Maestría en Ciencias de la Nutrición y los Alimentos, Universidad Autónoma Metropolitana, Unidad Xochimilco, México. mcvelaz@ \\ correo.xoc.uam.mx. ORCID: https://orcid.org/0000-0002-5206-2823 \\ 2 Licenciatura en Nutrición Humana y especialista en Nutrición Gerontológica, Universidad Autónoma Metropolitana, Unidad Xochimilco, \\ México. mcabrer@correo.xoc.uam.mx. ORCID: https://orcid.org/0000-0001-7013-5697 \\ 3 Doctorado en Ciencias Odontológicas. Universidad Autónoma Metropolitana, Unidad Xochimilco, México. meirigo@correo.xoc.uam. \\ mx. ORCID: https://orcid.org/000-0002-7766-8063
}

Recibido: 23/10/2020. Aprobado: 24/02/2020. Publicado: 25/03/2021

Velázquez-Alva MC, Cabrer Rosales MF, Irigoyen Camacho ME. Evaluación de la intervención Importancia de la nutrición en pacientes adultos mayores con infección por COVID-19. Rev. Fac. Nac. Salud Pública. 2021;39(2):e344210. DOI: https://doi. org/10.17533/udea.rfnsp.e344210

\section{Resumen}

La nutrición cumple un valioso papel ante la infección por el nuevo coronavirus 2019 (COVID-19), tanto para la prevención como para el tratamiento, y en particular en los pacientes adultos mayores, debido a que tienen un alto riesgo de desarrollar desnutrición, la cual podría desencadenar diversas complicaciones relacionadas a la enfermedad, sobre todo durante el periodo de hospitalización o en la unidad de cuidados intensivos. El manejo nutricional, además del manejo farmacológico, debe ser implementado de manera adecuada y oportuna por el personal de salud. El objetivo de la presente revisión es brindar información sobre la intervención nutricional para el manejo de personas adultas mayores con infección de COVID-19.

--------Palabras clave: adultos mayores, Covid-19, desnutrición, SARS-CoV-2, terapia nutricional.

\begin{abstract}
Nutrition plays a valuable role in the face of infection by the new coronavirus 2019 (COVID-19) both for prevention and treatment, particularly in older adult patients due to their increased risk of developing malnutrition, which could cause various complications related to COVID-19, especially during the hospitalization period or, where appropriate, in the Intensive Care Unit. Nutritional therapy must be considered
\end{abstract}

and implemented by health personnel in an appropriate way. The objective of this review is to provide relevant information regarding nutritional intervention in different clinical fields for the management of older adults with COVID-19 infection.

-Keywords: COVID-19, SARS-CoV-2, Aged, Malnutrition, Nutrition Therapy. 


\section{Resumo}

A nutrição cumpre um papel valioso diante da infecção pelo novo corona vírus 2019 (COVID-19), tanto para a prevenção como para o tratamento, particularmente nos pacientes idosos, devido ao alto risco de desenvolver desnutrição, a qual poderia desencadear complicações diversas relacionadas à enfermidade, principalmente durante o período de hospitalização ou nas unidades de cuidados intensivos. O cuidado nutricional, além do cuidado farmacológico, deve ser implementado de maneira adequada e oportuna pelo pessoal de saúde. O objetivo da presente revisão é oferecer informação sobre a intervenção nutricional para o cuidado de pessoas idosas infectadas com COVID-19.

-Palavras-chave: Idosos; COVID-19; desnutrição; SARS-CoV-2; terapia nutricional

\section{Introducción}

La enfermedad causada por el nuevo coronavirus 2019 (COVID-19) es actualmente el problema de salud pública más grave en todo el mundo [1]. Hasta el 21 de diciembre del 2020 se habían registrado 77315962 casos de COVID-19 a nivel mundial, y la mortalidad alcanzada se registró en 1701145 [2]. La acelerada propagación del COVID-19 ha exacerbado los problemas en los sistemas de salud a nivel mundial, lo que ha provocado situaciones complejas en el entorno económico y social [3], viéndose mayormente afectados los grupos de población de menores ingresos, baja escolaridad y de mayor edad con un pobre estado de salud [4]. La utilidad de conocer las estrategias de atención nutricional en el manejo terapéutico del paciente con COVID-19 en diferentes áreas clínicas recae en la optimización del uso de recursos, lo que podría impactar de forma positiva tanto en los pacientes como en los sistemas del cuidado de la salud [5].

La atención adecuada del enfermo dependerá de la severidad de la infección, la cual se puede dividir en cuatro fases, de acuerdo con las siguientes variables: área clínica, relación presión parcial de oxígeno arterial y fracción de oxígeno inspirado $\left(\mathrm{PaO}_{2} / \mathrm{FiO}_{2}\right)$, y signos y síntomas de la infección [6] (véase Tabla 1).
Durante estas cuatro fases, el tratamiento se acompaña de múltiples medicamentos: antivirales (hidrocloroquina, remdesivir), inmunosupresores (corticoesteroides, inhibidores de la interleucina-6 y de la Janus quinasa) y anticoagulantes [6].

Infortunadamente, poca atención se le ha brindado al manejo nutricional de la enfermedad por COVID-19. Una nutrición adecuada, completa, suficiente, equilibrada e inocua ayuda a mantener un sistema inmune saludable, que es esencial para la prevención y el manejo de las infecciones virales, específicamente la producida en la actualidad por el COVID-19 [7], lo que es particularmente importante en las personas adultas mayores. Así, el objetivo de la presente revisión es brindar información sobre la intervención nutricional para el manejo de personas adultas mayores con infección de COVID-19.

\section{Efecto de la alimentación en la inmunidad}

Mientras que la dieta "occidentalizada" — caracterizada por ser elevada en grasas saturadas e hidratos de carbono simples (azúcares), y baja en fibra, antioxidantes y ácidos grasos insaturados - se ha asociado con la disminución del sistema inmune adaptativo, lo que se traduce en una pobre respuesta ante agentes virales y en un aumento en la inmunidad innata, lo que favorece la inflamación

Tabla 1. Fases de severidad de la infección por coviD-19

\begin{tabular}{|c|c|c|c|c|}
\hline \multirow[t]{2}{*}{ Variables } & \multicolumn{4}{|c|}{ Severidad de la infección } \\
\hline & Fase 1 & Fase 2 & Fase 3 & Fase 4 \\
\hline Área clínica & Comunidad & Hospitalización & Hospitalización subintensiva & $\begin{array}{l}\text { Unidad de } \\
\text { Cuidados } \\
\text { Intensivos (UCI) }\end{array}$ \\
\hline $\mathrm{PaO}_{2} / \mathrm{Fi}_{0} 2$ & $>300$ & $300-200$ & $<200$ & \\
\hline Síntomas & $\begin{array}{c}\text { Tos seca, dolor de cabeza y } \\
\text { fiebre moderada }\end{array}$ & $\begin{array}{c}\text { Disnea y saturación } \\
\text { periférica de oxígeno } \\
\text { baja }\end{array}$ & $\begin{array}{l}\text { Síndrome de dificultad } \\
\text { respiratoria aguda (SDRA) e } \\
\text { insuficiencia cardíaca }\end{array}$ & $\begin{array}{l}\text { Trombosis } \\
\text { pulmonar }\end{array}$ \\
\hline Signos & $\begin{array}{l}\text { Linfopenia y aumento leve de: } \\
\text { - Tiempo de protrombina } \\
\text { - Dímero-D (marcador anormal } \\
\text { de coagulación) }\end{array}$ & $\begin{array}{l}\text { Imagen anormal } \\
\text { del tórax a nivel } \\
\text { pulmonar }\end{array}$ & $\begin{array}{l}\text { Concentraciones plasmáticas } \\
\text { elevadas de: } \\
\text { - Proteína C reactiva } \\
\text { - Lactato deshidrogenasa } \\
\text { - Troponina } \\
\text { - Interleucina-6 }\end{array}$ & $\begin{array}{l}\text { Aumento } \\
\text { anormal del } \\
\text { dímero-D }\end{array}$ \\
\hline
\end{tabular}

Fuente: adaptada de [6]. 
crónica, por su parte, un consumo aumentado de fibra, cereales integrales, grasas insaturadas y antioxidantes podría tener un efecto antiinflamatorio y, a largo plazo, relacionarse con la prevención de la obesidad y el mantenimiento de un peso adecuado, lo que incluso, en el futuro, podría mejorar la efectividad de las vacunas [8].

El apego a determinados patrones de alimentación, como la dieta mediterránea, caracterizada por su abundante aporte de frutas, verduras, leguminosas y cereales enteros, así como de cantidades moderadas de productos lácteos, aves, pescado y vino tinto, y un bajo aporte de carnes rojas, azúcares y alimentos procesados, podría tener un efecto positivo en pacientes con COVID-19, debido a su elevado contenido de vitaminas D, E, C, A,
$\mathrm{B}_{12} \mathrm{y}$ folatos, y de minerales como zinc, selenio, hierro, manganeso, así como de polifenoles [9].

Recientemente, se han identificado nutrimentos que promueven el funcionamiento del sistema inmunitario y se ha analizado su relación con indicadores epidemiológicos de COVID-19. En un estudio de diseño ecológico del 2020, se observó una asociación inversa entre la ingesta de vitaminas $\mathrm{D}, \mathrm{C}, \mathrm{B}_{12} \mathrm{y}$ hierro con una mayor incidencia o mortalidad de COVID-19, por lo que la biodisponibilidad de estos nutrimentos puede ser una herramienta fundamental para el fortalecimiento del sistema inmune [10]. En la Tabla 2 se muestran algunas de las fuentes alimentarias que contienen estos nutrimentos.

Tabla 2. Fuentes alimenticias de vitaminas y minerales claves en el funcionamiento del sistema inmune

\begin{tabular}{ll}
\hline \multicolumn{1}{c}{ Nutrimento } & \multicolumn{1}{c}{ Fuentes alimentarias } \\
\hline Vitamina $\mathrm{C}$ & Frutos rojos, cítricos, coliflor, repollo, pimiento, tomate, cebollín, perejil \\
Vitamina $\mathrm{D}$ & Aceite de hígado de bacalao, sardinas, atún, champiñones, yema de huevo \\
Vitamina $\mathrm{B}_{12}$ & Lácteos (leche y yogurt), huevos, carne, pescado, hígado \\
Hierro & Carnes rojas, leguminosas, espinacas, frutos secos, nueces \\
\hline
\end{tabular}

\section{Pacientes mayores de 60 años de edad}

Se ha reportado que las personas de edad avanzada tienen mayor riesgo de desarrollar la infección por COVID-19 de manera más grave, con un peor pronóstico, con un aumento en el riesgo de adquirir síndrome de dificultad respiratoria aguda (SDRA) y presentan mayor tasa de mortalidad [11-13].

La edad avanzada y las enfermedades múltiples y crónicas, como la diabetes mellitus tipo 2 (DMT2) y la enfermedad cardiovascular, se han asociado a un estado de nutrición deteriorado [13], en particular, entre adultos mayores frágiles, en quienes su pronóstico y recuperación, cuando contraen la infección por COVID-19, se afectan de forma negativa [14].

Recientemente, tanto en una revisión sistemática como en una investigación clínica, se identificaron distintas herramientas de tamizaje que permiten la evaluación de riesgo nutricional en personas adultas mayores con COVID-19, de las cuales resaltan las siguientes, por el valor predictivo que presentan:

- Evaluación mínima del estado de nutrición versión corta (Mini Nutritional Assessment ${ }^{\circledR}$ Short Form, MNA $\left.{ }^{\circledR}-\mathrm{SF}\right)$, predictiva para disminución del apetito y pérdida de peso [15].

- Tamizaje de riesgo nutricional - 2002 (Nutritional Risk Screening, NRS-2002), predictivo para la duración de la estancia hospitalaria [15].
- Índice de riesgo nutricional geriátrico (Geriatric Nutritional Risk Index, GNRI), predictivo para sobrevivencia intrahospitalaria [16].

- Escala modificada simplificada de riesgo nutricional en el paciente crítico (Modified Nutrition Risk in Critically, mNUTRIC), predictiva para los siguientes resultados adversos [15]:

- SDRA.

- Estado de choque.

- Infarto agudo al miocardio.

- Infección secundaria.

- Mortalidad.

Con respecto al tamizaje del riesgo nutricional, Li et $a l$, en un trabajo con un diseño de corte transversal, reportaron que los principales factores de riesgo independientes para desnutrición evaluada de MNA $®$ en ancianos hospitalizados con COVID-19 en Wuhan, China, fueron: DMT2, circunferencia de pantorrilla reducida y niveles plasmáticos disminuidos de albúmina [17].

Además, se ha sugerido que la infección por COVID-19 podría estar relacionada con la aparición de sarcopenia y caquexia, al estar asociada a pérdida de peso corporal y reducción de la masa muscular esquelética, lo que podría explicarse por la inmovilización prolongada observada en pacientes con ventilación mecánica. Así mismo, la anosmia y la pérdida del gusto se han asociado a niveles elevados de citocinas proinflamatorias, que a su vez son causas comunes de anorexia y de niveles bajos de albúmina [18]. 
Por otra parte, se identificó, a través de un estudio de cohorte retrospectivo, que la linfopenia es un factor pronóstico negativo en pacientes con COVID-19, mientras que los niveles plasmáticos de prealbúmina reducidos han sido de utilidad para predecir la progresión al SDRA [19].

Adicionalmente, se ha reportado que la obesidad se relaciona con un mal pronóstico en pacientes comórbidos con COVID-19 [20]. Particularmente en México, se ha identificado un mayor riesgo tanto para hospitalización como para el ingreso a la UCI y apoyo con ventilación mecánica entre adultos mayores con obesidad e insuficiencia renal, enfermedad pulmonar obstructiva crónica y DMT2 [21].

\section{Recomendaciones nutricionales para pacientes adultos mayores hospitaliza- das por COVID-19}

El momento del soporte nutricional es crítico, debido a la rápida progresión de la gravedad de la infección por COVID-19 en pacientes de mayor edad [13]. Durante la hospitalización de las personas adultas mayores es fundamental considerar y atender la comorbilidad, así como los efectos no deseados de la hospitalización (véase Figura 1).

Se ha descrito que el reposo en cama por hospitalización se asocia a los siguientes efectos adversos: disminución en la masa muscular (sarcopenia), pérdida de la fuerza muscular (dinapenia), deterioro en el desempeño físico y reducción de la capacidad aeróbica, lo que po-

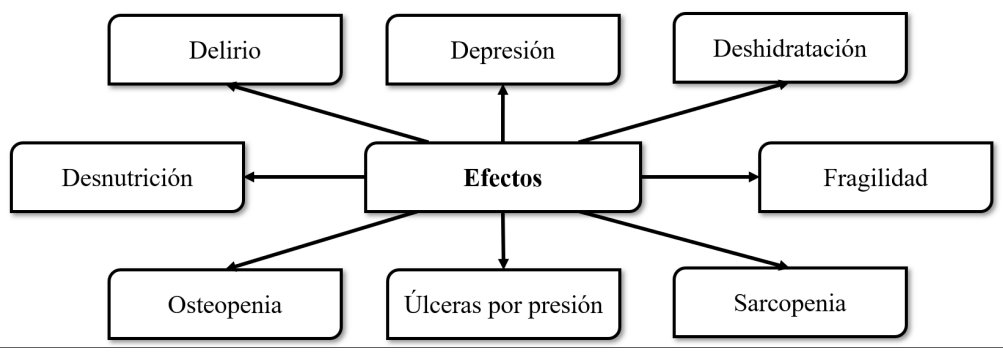

Figura 1. Efectos no deseados de la hospitalización en pacientes adultos mayores

dría comprometer la autonomía de los adultos mayores con COVID-19 [22,23].

Por lo tanto, la intervención nutricional complementaria al manejo farmacológico es sin duda muy importante entre los pacientes en riesgo de desnutrición, por lo que los protocolos estandarizados de apoyo nutricio son de gran utilidad en este momento [24].

Al respecto, el "Protocolo pragmático para la administración de terapia nutricional en pacientes pre-UCI con COVID-19" fue uno de los primeros en publicarse frente a la crisis de COVID-19 que se vivió en la región de Lombardía, en Italia (véase Figura 2) [25].

Por otro lado, la Sociedad Europea de Nutrición Enteral y Parenteral (ESPEN) publicó la "Declaración de expertos de ESPEN y orientación práctica para el manejo nutricional de personas con infección por SARS-CoV-2 [severe acute respiratory syndrome coronaviruses]", en quienes califican como pacientes de UCI, de edad avanzada, con polimorbilidad y desnutrición [26]. En la Tabla 3 se observan los valores allí recomendados para cubrir el requerimiento energético y de sustratos para los pacientes infectados COVID-19 [26].

En la declaración citada se recomienda que en pacientes en riesgo o infectados con SARS-CoV-2 se inicie el apoyo nutricional a través de la vía oral, mediante el uso de fórmulas especializadas, para cubrir tanto sus requerimientos de energía como de macro y micronutrientes. En aquellos pacientes que no alcancen a cubrir dichos requerimientos por la vía oral, se recomienda el uso de la nutrición enteral (NE), o bien, nutrición parenteral (NPT) cuando la NE no esté indicada o sea insuficiente [26].

Particularmente, entre los pacientes intubados en UCI, se recomienda que la administración de la NE sea por una sonda nasogástrica; la alimentación pospilórica estará indicada en pacientes con intolerancia gástrica después de tratamiento procinético o en aquellos con elevado riesgo de broncoaspiración. Se aconseja que una vez que el paciente este recibiendo NE, se le coloque en la posición prono, al ser más segura [26].

En algunas ocasiones, en pacientes con síntomas gastrointestinales como vómito y diarrea, así como con hipoxemia (potencialmente mortal), el inicio de la NE podría estar contraindicado o verse retrasado [29]; en aquellos pacientes que no toleren NE durante la primera semana en la UCI, el uso de NPT deberá ser valorado [26].

El inicio temprano de NPT deberá ser valorado en pacientes con desnutrición moderada y severa, así como en aquellos con choque séptico que requieren dosis 
elevadas de vasopresores y en enfermos que presenten síndromes gastrointestinales, para ayudar a minimizar el riesgo de isquemia intestinal. El monitoreo de la administración de la NPT es necesario para evitar complicaciones asociadas (infección del catéter, hiperglucemia, alteraciones hidroelectrolíticas) [28].

Se recomiendan alimentos modificados en textura tras extubación en pacientes con disfagia. Si la deglución no es segura, deberá continuarse NE [26].

Al inicio del soporte nutricio se debe considerar el riesgo de desarrollar el síndrome de realimentación (SRA), que consiste en una reacción anabólica causada por la terapia nutricional, que suele presentarse en los primeros tres días de su inicio y que se asocia a un desequilibrio de líquidos y electrolitos séricos o síntomas clínicos resultantes de cambios metabólicos. El SRA es una afección fisiológica potencialmente mortal, que se presenta en pacientes con desnutrición severa, enfermedad catabólica o en personas adultas mayores con síndrome de fragilidad, acompañado de comorbilidades [30,31]. Por esto, se recomienda cubrir los requerimientos nutricionales de forma gradual, sobre todo en los pacientes ancianos con multimorbilidad [26].

En pacientes que presenten SDRA se ha recomendado el uso de fórmulas enterales adicionadas con agentes antioxidantes, ácido eicosapentaenoico ( $\omega-3)$ y ácido gamma-linolénico $(\omega-6)$, ya que se ha demostrado que ofrecen beneficios en la oxigenación y disminución en los días de ventilación mecánica; no obstante, aún no se ha determinado si la neumonía por COVID-19 responde a estos nutrimentos [32].

Los antioxidantes (vitamina $\mathrm{C}$, polifenoles, epigalocatequina-3-galato y flavonoides) podrían cumplir un papel importante ante el estrés oxidativo en los pulmones, actuando como moduladores inmunes y mediadores de inflamación, por lo que su suplementación podría implementarse en pacientes con COVID-19 [32]. En un estudio de tipo observacional, se reportaron niveles bajos de vitamina $\mathrm{C}$ en pacientes críticamente enfermos, en quienes se pueden requerir dosis de hasta de 2-3 g / día [33]; no obstante, la evidencia para su uso en pacientes con COVID-19 es insuficiente [34].

Por otra parte, se ha hipotetizado que los ácidos grasos $\omega-3$ y la adiponectina podrían reducir la respuesta inflamatoria pulmonar, efecto benéfico posiblemente mediado por citocinas proinflamatorias [32]; sin embargo, aún son necesarios ensayos aleatorizados y controlados que sustenten la efectividad de suplementación con ácidos grasos $\omega-3$ en pacientes con SARS-CoV-2 [35].

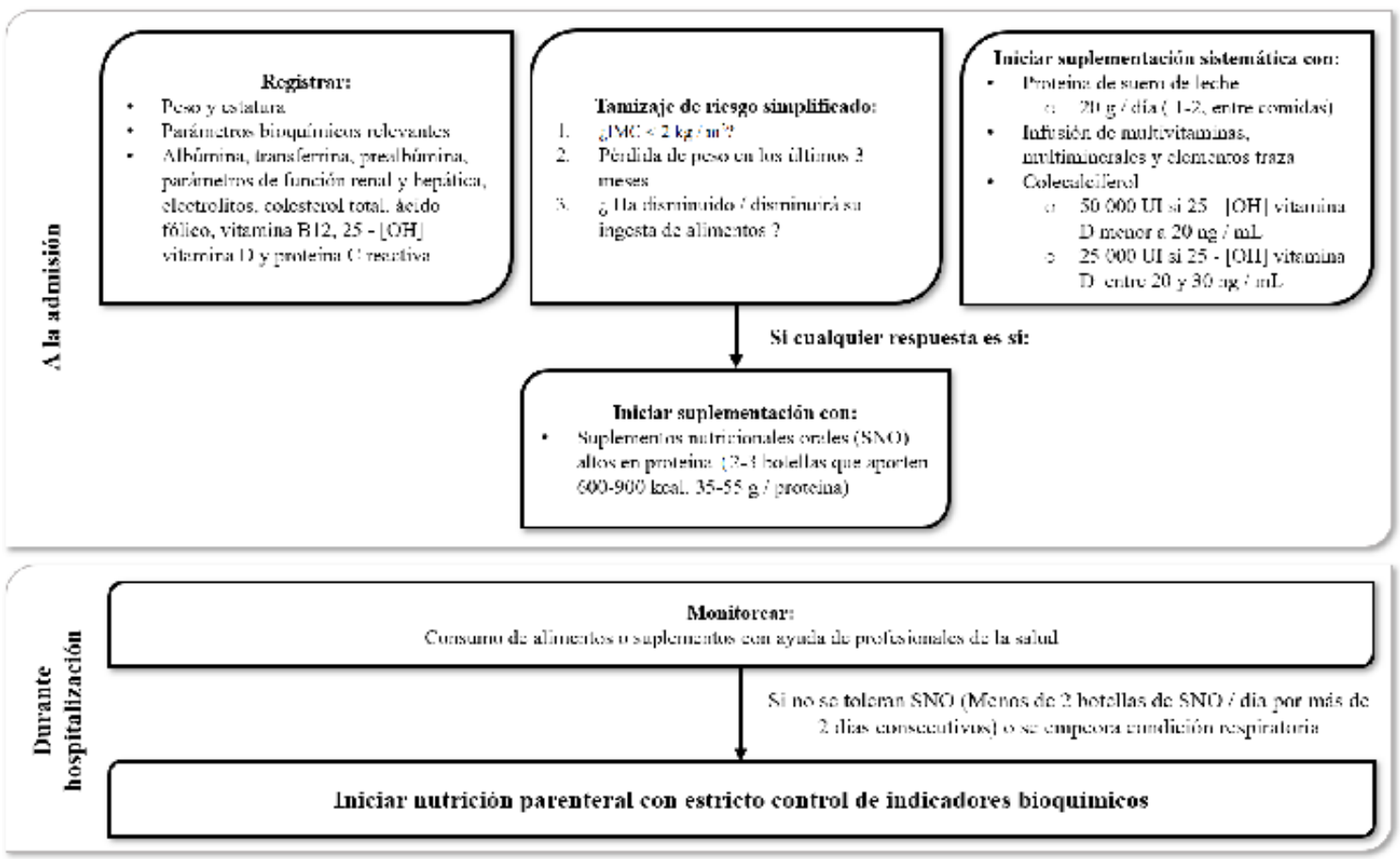

Figura 2. Protocolo pragmático para la administración de terapia nutricional en pacientes pre-UCl con covID-19. 25 - [OH] vitamina D: 25 hidroxivitamina D; IMc: Índice de masa corporal; uI: Unidades internacionales.

Fuente: adaptada de [25]. 
Tabla 3. Recomendaciones nutricionales de energía, proteína, hidratos de carbono, lípidos, vitaminas y elementos traza en pacientes con Covid-19

\begin{tabular}{ll}
\hline \multicolumn{1}{c}{ Nutrimento } & \multicolumn{1}{c}{ Recomendación } \\
\hline Energía & $27 \mathrm{kcal} / \mathrm{kg} /$ día en pacientes con polimorbilidad mayores de 65 años \\
& $30 \mathrm{kcal} / \mathrm{kg} /$ día en pacientes polimórbidos severamente desnutridos (considerar riesgo de \\
& síndrome de realimentación, por lo que esta meta deberá alcanzarse de manera paulatina) \\
& $1,0 \mathrm{~g} / \mathrm{kg} /$ día en personas adultas mayores \\
& $\geq 1,0 \mathrm{~g}$ en pacientes polimórbidos \\
Proteína & Hasta $2 \mathrm{~g} / \mathrm{kg} /$ día en pacientes con enfermedad severa o desnutrición [27,28] \\
Hidratos de carbono y lípidos & $\begin{array}{l}\text { Su aporte deberá adaptarse al requerimiento energético y considerar una proporción de } \\
\text { lípidos e hidratos de carbono entre 30:70 (en pacientes sin insuficiencia respiratoria) y 50:50 } \\
\text { (en pacientes con ventilación mecánica) }\end{array}$ \\
& $\begin{array}{l}\text { Suplementación o suministro diario de vitaminas y elementos para cubrir la ingesta diaria } \\
\text { Vecomendada }\end{array}$ \\
\hline
\end{tabular}

Fuente: adaptada de [26].

\section{Conclusión}

La pandemia por COVID-19 es un riesgo para la salud pública mundial, por su incesante propagación, por lo que las estrategias que permitan optimizar los recursos médicos son de utilidad para reducir la carga a los sistemas de la salud en la mayoría de los países, en particular, a nivel hospitalario, donde las guías específicas de apoyo nutricional para el manejo de los pacientes con COVID-19 deben ser consideradas y aplicadas.

El uso de suplementos alimenticios en etapas tempranas de la hospitalización es recomendable. Se debe prestar especial atención al adulto mayor, por su elevado riesgo de complicaciones y mortalidad frente a la infección por COVID-19.

\section{Declaración de responsabilidad}

Las autoras declaran que la información contenida en el presente manuscrito es de su autoría.

\section{Financiación}

No se recibió ninguna fuente de financiación.

\section{Conflicto de interés}

No se tiene conflicto de interés por parte de ninguna de las autoras.

\section{Declaración de autoría}

Las tres autoras declaran que participaron en la conceptualización, la revisión de la literatura, el diseño, la escritura y la edición final del manuscrito.

\section{Referencias}

1. World Health Organization. Coronavirus disease (COvID-19): Situation report, 206 [internet]. 2020 [citado 2020 oct. 12]. Disponible en: https://apps.who.int/iris/handle/10665/333839?localeattribute $=$ es\&

2. Johns Hopkins University. Covid-19 Dashboard by the Center for Systems Science and Engineering (CSSE) at Johns Hopkins University. [internet]. 2020 [citado 2020 dic. 20]. Disponible en: https://gisanddata.maps.arcgis.com/apps/opsdashboard/index. html\#/bda7594740fd40299423467b48e9ecf6

3. Cheema S, Ameduri M, Abraham A, et al. The Covid-19 pandemic: The public health reality. Epidemiology \& Infection. 2020;148:1-4. Dor: https://doi.org/10.1017/S0950268820002216

4. Irigoyen-Camacho ME, Velazquez-Alva MC, Zepeda-Zepeda MA, et al. Effect of income level and perception of susceptibility and severity of CoviD-19 on stay-at-home preventive behavior in a group of older adults in Mexico City. Int. J. Environ. Res. Public Health. 2020;17(20):1-16. DoI: https://doi.org/10.3390/ ijerph17207418

5. Naja F, Hamadeh R. Nutrition amid the Covid-19 pandemic: Amulti-level framework for action. Eur J Clin Nutr. 2020;74(8):111721. DoI: https://doi.org/10.1038/s41430-020-0634-3

6. Lauretani F, Ravazzoni G, Federica Roberti M, et al. Assessment and treatment of older individuals with COvID-19 multi-system disease: Clinical and ethical implications. Acta Biomedica. 2020;91(2):150-68. DoI: https://doi.org/10.23750/abm. v91i2.9629

7. Kalea AZ, Klimis-Zacas D. Editorial: Challenges and Insights amidst the Covid-19 pandemic: Nutrition, the immune system and disease risk. Current opinion in clinical nutrition and metabolic care. 2020;23(4):233-5. DoI: https://doi.org/10.1097/ MCO.0000000000000667

8. Butler MJ, Barrientos RM. The impact of nutrition on Covid-19 susceptibility and long-term consequences. Brain Behav Immun. 2020;87:53-4. Dor: https://doi.org/10.1016/j.bbi.2020.04.040

9. Galmés S, Serra F, Palou A. Current state of evidence: Influence of nutritional and nutrigenetic factors on immunity in the COVID-19 pandemic framework. Nutrients. 2020;12(9):1-33. DOI: https://doi.org/10.3390/nu12092738 
10. Trujillo-Mayol I, Guerra-Valle M, Casas-Forero N, et al. Western dietary pattern antioxidant intakes and oxidative stress: Importance during the SARS-CoV-2/COVID-19 pandemic. Adv Nutr. 2021; 12(3): 670-681. DOI: https://doi.org/10.1093/advances/nmaa171

11. Zheng Z, Peng F, Xu B, et al. Risk factors of critical \& mortal COVID-19 cases: A systematic literature review and meta-analysis. J Infect. 2020;81(2):e16-25. DOI: https://doi.org/10.1016/j. jinf.2020.04.021

12. Liu K, Chen Y, et al. Clinical features of COVID-19 in elderly patients: A comparison with young and middle-aged patients. Journal of Infection. 2020;80(6):e14-8. Dor: https://doi.org/10.1016/j. jinf.2020.03.005

13. Zhou F, Yu T, Du R, et al. Clinical course and risk factors for mortality of adult inpatients with COVID-19 in Wuhan, China: A retrospective cohort study. The Lancet. 2020;395(10229):105462. DoI: https://doi.org/10.1016/S0140-6736(20)30566-3

14. Azzolino D, Saporiti E, et al. Nutritional considerations in frail older patients with COVID-19. J Nutr Health Aging. 2020;24(7):6968. DOI: https://doi.org/10.1007/s12603-020-1400-x

15. Silva DFO, Lima SCVC, Sena-Evangelista KCM, et al. Nutritional risk screening tools for older adults with CovID-19: A systematic review. Nutrients. 2020;12(10):2956. DoI: https://doi. org/10.3390/nu12102956

16. Recinella G, Marasco G, Serafini G, et al. Prognostic role of nutritional status in elderly patients hospitalized for COVID-19: A monocentric study. Aging Clin Exp Res. 2020;32(2):2695-701. DOI: https://doi.org/10.1007/s40520-020-01727-5

17. Li T, Zhang Y, Gong C, et al. Prevalence of malnutrition and analysis of related factors in elderly patients with CovID-19 in Wuhan, China. Eur J Clin Nutr. 2020;74(6):871-5. Dor: https:// doi.org/10.1038/s41430-020-0642-3

18. Morley JE, Kalantar-Zadeh K, Anker SD. CovID-19: A major cause of cachexia and sarcopenia? J Cachexia Sarcopenia Muscle. 2020 Aug 1;11(4):863-5. DoI: https://doi.org/10.1002/jcsm.12589

19. Wu C, Chen X, Cai Y, et al. Risk factors associated with acute respiratory distress syndrome and death in patients with coronavirus disease 2019 pneumonia in Wuhan, China. JAMA Internal Medicine. 2020;180(7):934-43. DoI: https://doi.org/10.1001/jamainternmed.2020.0994

20. Petrova D, Salamanca-Fernández E, Rodríguez Barranco M, et al. Obesity as a risk factor in COVID-19: Possible mechanisms and implications. Aten Primaria. 2020;52(7):496-500. DoI: https://doi. org/10.1016/j.aprim.2020.05.003

21. Bello-Chavolla OY, González-Díaz A, Antonio-Villa NE, et al. Unequal impact of structural health determinants and comorbidity on COVID-19 severity and lethality in older Mexican adults: Considerations beyond chronological aging. J Gerontol. 2020;76(3): e52-e59. DoI: https://doi.org/10.1093/gerona/glaa163

22. Coker RH, Hays NP, Williams RH, et al. Bed rest promotes reductions in walking speed, functional parameters, and aerobic fitness in older, healthy adults. J Gerontol A Biol Sci Med Sci. 2015;70(1):91-6. DoI: https://doi.org/10.1093/gerona/glu123
23. Kortebein P, Symons TB, Ferrando A, et al. Functional impact of 10 days of bed rest in healthy older adults. J Gerontol. 2008;63A(10):1076-81. DOI: https://doi.org/10.1093/gerona/63.10.1076

24. Aprahamian I, Cesari M. Geriatric syndromes and SARs-CoV-2: More than just being old. J Frailty Aging. 2020;9(3):127-9. DoI: https://doi.org/10.14283/jfa.2020.17

25. Caccialanza R, Laviano A, Lobascio F, et al. Early nutritional supplementation in non-critically ill patients hospitalized for the 2019 novel coronavirus disease (COVID-19): Rationale and feasibility of a shared pragmatic protocol. Nutrition. 2020;74:110835. DOI: https://doi.org/10.1016/j.nut.2020.110835

26. Barazzoni R, Bischoff SC, Breda J, et al. ESPEN expert statements and practical guidance for nutritional management of individuals with SARS-CoV-2 infection. Clinical Nutrition. 2020;39(6):1631-8. DOI: https://doi.org/10.1016/j.clnu.2020.03.022

27. Bauer J, Biolo G, Cederholm T, et al. Evidence-based recommendations for optimal dietary protein intake in older people: A position paper from the prot-age study group. Am Med Dir Assoc. 2013;14(8):542-59. DOI: https://doi.org/10.1016/j.jamda.2013.05.021

28. Martindale R, Patel JJ, Taylor B, et al. Nutrition Therapy in the Patient with COVID-19 Disease Requiring ICU Care [internet]. 2020 [citado 2020 dic. 20]. Disponible en: https://www.sccm.org/ COVID19RapidResources/Resources/Nutrition-Therapy-in-thePatient-with-COVID-19-Dis

29. Laviano A, Koverech A, Zanetti M. Nutrition support in the time of SARS-CoV-2 (COVID-19). Nutrition. 2020;74:110836. DOI: https://doi.org/10.1016/j.nut.2020.110834

30. Aubry E, Friedli N, et al. Refeeding syndrome in the frail elderly population: Prevention, diagnosis and management. Clin Exp Gastroenterol. 2018;11:255-64. DoI: https://doi.org/10.2147/ CEG.S136429

31. Friedli N, Stanga Z, Culkin A, et al. Management and prevention of refeeding syndrome in medical inpatients: An evidence-based and consensus-supported algorithm. Nutrition. 2018;47:13-20. DOI: https://doi.org/10.1016/j.nut.2017.09.007

32. Messina G, Polito R, Monda V, et al. Functional role of dietary intervention to improve the outcome of COVID-19: A hypothesis of work. Int J Mol Sci. 2020;21(9):3104. DoI: https://doi. org/10.3390/ijms21093104

33. Carr AC, Rosengrave PC, Bayer S, et al. Hypovitaminosis C and vitamin $\mathrm{C}$ deficiency in critically ill patients despite recommended enteral and parenteral intakes. Crit Care. 2017;21(1):300. DoI: https://doi.org/10.1186/s13054-017-1891-y

34. National Institutes of Medicine. COVID-19 treatment guidelines: Vitamin C. National Institutes of Medicine [internet]. 2020 [citado 2020 dic. 20]. Disponible en: https://www.covid19treatmentguidelines.nih.gov/adjunctive-therapy/vitamin-c/

35. Rogero MM, Leão M de C, Santana TM, et al. Potential benefits and risks of omega-3 fatty acids supplementation to patients with COVID-19. Free Radic. Biol. Med. 2020;156:190-9. Dor: https:// doi.org/10.1016/j.freeradbiomed.2020.07.005 